\title{
Development of adenovirus immobilization strategies for in situ gene therapy
}

\author{
Wei-Wen $\mathrm{Hu}^{1,2}$ \\ Michael W. Lang ${ }^{2}$ \\ Paul H. Krebsbach ${ }^{1,2 *}$ \\ ${ }^{1}$ Department of Biomedical \\ Engineering, University of Michigan, \\ Ann Arbor, MI, USA \\ ${ }^{2}$ Department of Biologic and \\ Materials Sciences, School of \\ Dentistry, University of Michigan, \\ Ann Arbor, MI, USA \\ *Correspondence to: \\ Paul H. Krebsbach, Department of \\ Biologic and Materials Sciences, \\ School of Dentistry, K1030, 1011 \\ North University Avenue, University \\ of Michigan, Ann Arbor, MI \\ 48109-1078, USA. \\ E-mail:paulk@umich.edu
}

\begin{abstract}
Background Regenerative gene therapy using viral vectors enables transduced cells to express bioactive factors in vivo. Viral delivery with spatial control can enhance transduction efficiency and may limit systemic infection. Consequently, we tethered biotinylated adenovirus via interactions with avidin on chitosan surfaces to gain robust control for in situ transduction.

Methods Avidin was either directly conjugated to chitosan (virus-biotinavidin-material; VBAM) or indirectly docked on biotinylated chitosan surfaces (virus-biotin-avidin-biotin-material; VBABM) to tether biotinylated adenovirus. Enzyme-linked immunosorbent assay (ELISA) and spectroscopic analysis were performed to demonstrate the binding profiles. Biotinalkaline phosphatase and biotinylated adenovirus were used as different sized particles to evaluate binding efficiencies and were compared by the Sips isotherm adsorption method. Scanning electron microscopy (SEM) examination illustrated virus distribution, and the transduction efficiency was determined by in vitro cell transduction.
\end{abstract}

Results ELISA and spectroscopic analysis both demonstrated that the VBAM system led to multilayer avidin formation on biomaterial surfaces, whereas VBABM formed a monolayer of avidin. Sips isotherm adsorption indicated that the VBAM method increased heterogeneity and steric hindrance of binding sites. By contrast, the VBABM method docked avidin on chitosan surfaces and orientated the binding sites to facilitate ligand binding. In addition, SEM images illustrated that the VBABM method led to more even viral distribution. In vitro cell infection experiments also demonstrated that the VBABM system enhanced virus immobilization and thus improved cell transduction efficiency over the VBAM system.

Conclusions The VBABM strategy is a superior method for in situ transduction from biomaterials. This strategy could be adapted for use with a variety of biomaterials as well as viral vectors, and thus may be an alternative method for in vivo regenerative gene therapy. Copyright (c) 2008 John Wiley \& Sons, Ltd.

Keywords adenovirus; avidin/biotin; chitosan; gene delivery; surface modification; tissue engineering

\section{Introduction}

Received: 24 January 2008

Revised: 26 March 2008

Accepted: 2 June 2008
Regenerative gene therapy by viral vectors can transduce cells to sustainedly and stably express bioactive factors in vivo. Several viral delivery methods have been developed for regenerative gene therapy. Bolus delivery by direct 
injection or inhalation is the easiest viral delivery method. However, high dosages are necessary for therapeutic effects because the virus may diffuse from the area of injection, which may limit the regional effectiveness and induce an immune response [1-4]. In addition, the poor spatial control in bolus delivery may cause unintended systemic infection [5]. Polymeric release is an improved method that incorporates viral vectors in a polymer matrix to control release by polymer degradation [6]. This method permits the sustained release of the virus to reduce viral immunogenicity and limit virus degradation [6,7]. Unfortunately, because the virus is not easily localized at the desired therapeutic target site using this method, unwanted transduction at distant sites may result in unanticipated consequences [8].

Substrate-mediated viral delivery is an alternative approach that functions by complexing virus in or on a biomaterial that also serves as a substrate for cell adhesion [8,9]. Using this strategy, lyophilized viral vectors within scaffolds can improve transduction efficiency, and thus the dose of virus administrated can be reduced [10]. However, the diffusion of lyophilized virus from a material surface is still unavoidable in this method, and therefore spatial control is limited. Because viral vectors immobilized on a biomaterial scaffold can transduce cells in situ to reduce possible risks during in vivo application, we exploited this strategy to gain robust control of cell transduction from chitosan as a test material for the long term goal of regenerative gene therapy.

Chitosan is a biodegradable polysaccharide derived from crustacean shells [11]. The nontoxic and tissue compatible properties of chitosan support its use as a biomaterial for pharmaceutical and drug delivery research $[12,13]$. In addition, chitosan has a hydrophilic surface that may promote cell adhesion, proliferation, and differentiation, and thus is broadly used as scaffold material $[14,15]$. Furthermore, chitosan is synthesized by chitin deacetylation due to its ambient amines, and can be easily modified [16]. Therefore, we used chitosan as a carrier with its active functional groups to immobilize adenovirus on its surface and investigated its potential to effectively deliver bioactive virus.

The binding forces involved in directly conjugating a virus on a biomaterial surface may be too strong to allow for the efficient release of virus for cell internalization. Therefore, bioconjugation mediated by noncovalent bonding should be a more effective method of immobilizing viral particles on material surfaces for in situ transduction. Virus can be immobilized by antibody binding to localize gene expression on substrates and avoid diffusion for in vivo transduction [17-19]. However, because an antibody is specific to an antigen, different viral vectors would need to be captured by different antibodies. In addition, because the titer of antibody is highly affected by the host animal, a stable source of antibody may be a problem. Furthermore, immunization is expensive and time consuming. These drawbacks make antibody immobilization difficult to apply as a universal viral delivery method for clinical application.

The biotin-avidin interaction is known to be the strongest noncovalent bond, and this system has been used for biotechnology applications [20]. The molecules are commercially available and can be conjugated with different materials. For example, chitosan has been successfully biotinylated for enzyme immobilization as bioprobes [21], and adenovirus biotinylation has been applied to cell targeting and virus purification methods [22]. In addition, biotinylated virus has been immobilized on avidin coated culture plates for in situ infection [23]. However, protein coating by physical adsorption may be less stable in in vivo environments [24].

Consequently, two different avidin immobilization strategies on material surfaces were developed for this study. Avidin was either directly conjugated to chitosan (virus-biotin-avidin-material; VBAM) or indirectly docked on biotinylated chitosan surfaces (virus-biotin-avidin-biotin-material; VBABM) to tether biotinylated adenovirus. By a range of experimental analyses, we determined an effective and universal viral delivery model for in situ transduction.

\section{Materials and methods \\ Adenovirus biotinylation and quantification}

Adenovirus encoding the $\beta$-galactosidase gene and localization sequence (AdLacZ) was prepared by the Vector Core, University of Michigan. This adenovirus was biotinylated by sulfosuccinimidyl-6-[biotinamido] hexanoate (SulfoNHS-LC-Biotin) (Pierce, Rockford, IL, USA) which was dissolved in phosphate-buffered saline (PBS; Pierce) before being reacted with adenovirus. The conjugation reaction was performed at $4{ }^{\circ} \mathrm{C}$ for $2 \mathrm{~h}$, and then quenched by an equal volume $1 \mathrm{M}$ glycin (SigmaAldrich, St Louis, MO, USA) in PBS. Ultrafiltration was applied using centricon filters (50 kDa MWCO; Millipore, Billerica, MA, USA) to remove unreacted biotin. The biotinylated virus was sterilized by passing through a $0.2 \mu \mathrm{m}$ syringe filter (Nalgene, Rochester, NY, USA).

To determine an appropriate concentration of SulfoNHS-LC-Biotin for virus biotinylation, an avidin conjugated alkaline phosphatase (avidin-AP; MP Biomedicals, Aurora, OH, USA) sandwich enzyme-linked immunosorbent assay (ELISA) was used to detect biotin on viral surfaces: Goat anti-adenovirus antibody ( $1 \mu \mathrm{g} /$ well) (Abcam, Cambridge, MA, USA) was coated on 96 -well plates (Coring, Lowell, MA, USA) at $4{ }^{\circ} \mathrm{C}$ overnight, which was then blocked with $1 \%$ bovine serum albumin (BSA; Roach, Indianapolis, IN, USA) in PBS. Biotinylated virus was added $100 \mu \mathrm{l} /$ well and incubated at room temperature for $1 \mathrm{~h}$, and the plates were washed with $0.05 \%$ Tween-20 in PBS (PBST; Teknova, Hollister, CA, USA). Subsequently, avidin-AP was added to label the biotin on the plate for 
1 h. After three washes in PBST, substrate $p$-nitrophenyl phosphate (PNPP; Pierce) was added for $20 \mathrm{~min}$, and enzyme activity was detected by optical density at $405 \mathrm{~nm}$ $\left(\mathrm{OD}_{405}\right)$.

The number of biotin molecules per adenovirus was determined by a 2-(4'-hydroxyazobenzene) benzoic acid (HABA; Pierce) assay: $6 \mu \mathrm{mol}$ of HABA was added to $5 \mathrm{mg}$ of avidin in $10 \mathrm{ml}$ of PBS to prepare the HABA/avidin solution. The solution $(0.9 \mathrm{ml})$ was transferred by pipette into a $1 \mathrm{ml}$ cuvette to read spectrophotometrically at $\mathrm{OD}_{500}$ (Beckman Coulter, Fullerton, CA, USA). Biotinylated virus $(0.1 \mathrm{ml})$ was then added and mixed in the cuvette to read $\mathrm{OD}_{500}$. HABA had an absorption wavelength of $500 \mathrm{~nm}$ when added to avidin. However, this absorption decreased proportionally when biotin was added. This was because the biotin displaced the HABA dye due to its higher affinity for avidin. Therefore, the biotinylation degree was calculated using the formula:

$$
\frac{\text { Biotin }}{\text { Virus Particles }}=\frac{\frac{\Delta O D_{500 \mathrm{~nm}}}{34000} \times 10}{\text { mmoles Virus Particles } / \mathrm{ml}}
$$

where $\Delta \mathrm{OD}_{500}=\mathrm{OD}_{500 \mathrm{~nm}}$ of $\mathrm{HABA} /$ avidin $\times 0.9-$ $\mathrm{OD}_{500 \mathrm{~nm}}$ of HABA/avidin/biotintinylated virus.

To determine whether biotinylation affected the bioactivity of adenovirus, the fibroblast cell line C4 (ATCC, Manassas, VA, USA) was cultured in alphaminimal essential medium (Gibco, Carlsbad, CA, USA) containing 10\% fetal bovine serum (Gibco) and penicillin (100 units/ml)-streptomycin (100 mg/ml) (Gibco) at a density of $5 \times 10^{4}$ cells/well in 24-well culture plates (Coring) for 1 day. Subsequently, AdLacZ (with or without biotinylation) was added to the culture wells for $48 \mathrm{~h}$ of infection. The transduction efficiencies of each group were determined by the expression of $\beta$-galactosidase, which was detected using a sandwich ELISA kit (Roach).

\section{Chitosan film preparation}

Chitosan with molecular weight in the range 100$300 \mathrm{kDa}$ (Acros, Geel, Belgium) was dissolved in $0.5 \mathrm{M}$ acetic acid for a final concentration of $1.5 \%$. After melting at $60^{\circ} \mathrm{C}$ overnight, the chitosan solution was filtered through a $0.8-\mu \mathrm{m}$ membrane. The chitosan solution ( $1 \mathrm{ml} /$ well) was placed into 24 -well culture plates that were then incubated at $80^{\circ} \mathrm{C}$ overnight to evaporate the acetic acid solvent. Each well was neutralized in $0.3 \mathrm{M}$ $\mathrm{NaOH}$ for 30 min and was then washed with PBS.

\section{Avidin conjugation}

Avidin (Pierce) in PBS was placed in chitosan-coated wells ( $0.25 \mathrm{ml}$ /well). Glutaraldehyde (Acros), the homobifunctional crosslinker for bioconjugation, was also diluted in PBS then added at $0.25 \mathrm{ml} /$ well. After $2 \mathrm{~h}$, the wells were washed with PBS to remove nonreactive reagent. Glycine
(2 M) in PBS was used to quench nonreactive crosslinker on the plate for $30 \mathrm{~min}$. Finally, the plates were washed with $70 \%$ ethanol for sterilization.

For the VBABM method, avidin was indirectly docked on a biotinylated surface before immobilizing biotinylated molecules. Avidin was dissolved in PBS and placed at $0.25 \mathrm{ml} /$ well for $2 \mathrm{~h}$ of incubation at room temperature and then washed with PBS to remove excess avidin.

The surface avidin was evaluated by a biotin conjugated alkaline phosphatase (biotin-AP; Pierce) assay: After blocking with $1 \%$ BSA-PBS, biotin-AP was diluted in PBS $(12 \mathrm{ng} / \mathrm{ml})$ and added to the plates $(0.25 \mathrm{ml} /$ well $)$ for $1 \mathrm{~h}$, and was developed by $0.2 \mathrm{ml}$ of PNPP substrate for $20 \mathrm{~min}$ before being read spectrophotometrically at $\mathrm{OD}_{405}$.

\section{Biotin conjugation}

(+)-Biotinyl-3,6,9-trioxaundecanediamine (Amine- $\mathrm{PEO}_{3}$ Biotin; Pierce) was dissolved in PBS and placed in chitosan-coated wells at $0.25 \mathrm{ml} /$ well. Glutaraldehyde was also diluted in PBS and then added at $0.25 \mathrm{ml} /$ well. After $2 \mathrm{~h}$ of incubation at room temperature, the wells were washed with PBS to remove nonreactive regent. Quenching by glycine solution and sterilizing by $70 \%$ ethanol were performed as previously described.

Surface biotin was quantified by avidin-AP. After blocking with 1\% BSA-PBS, avidin-AP was diluted in PBS $(0.112 \mu \mathrm{g} / \mathrm{ml})$ and added at $0.25 \mathrm{ml} /$ well to incubate for $1 \mathrm{~h}$, and was then detected by substrate PNPP, as previously described.

\section{Ultraviolet (UV) detection for avidin immobilization quantification}

Avidin has a maximum absorption at a wavelength of $230 \mathrm{~nm}$, which was determined by a scanning spectrum. Therefore, a UV spectrometer (Biotek, Winooski, VT, USA) was used to evaluate the total amount of avidin conjugation. After avidin conjugated with glutaraldehyde on the material surface, $150 \mu \mathrm{l}$ of supernatant was transferred to UV-penetrateable 96-microwell plates (Coring). Standard avidin solutions with different concentrations were used for comparison. The immobilized avidin was determined by subtracting the supernatant avidin values from the total amount of avidin present before the reaction.

\section{Biotin-AP immobilization assay}

Biotin-AP was used to simulate small biotinylated molecule binding profiles by two different avidin immobilization strategies. The surface biotin binding sites of the VBAM and VBABM systems were analysed by using different concentrations of biotin-AP. After $1 \mathrm{~h}$ of incubation, unbound biotin-AP was removed by washing with PBS. Finally, the PNPP substrate was developed for 5 min and absorbance was read at $\mathrm{OD}_{405}$. 


\section{Virus immobilization and sandwich ELISA assay}

The biotinylated AdLacZ was immobilized on the material surface by two strategies, the VBAM system and the VBABM system. After avidin immobilization, biotinylated virus was incubated on the chitosan surfaces for $2 \mathrm{~h}$ at $4^{\circ} \mathrm{C}$, and was then washed with PBS to remove unbound virus.

The immobilized virus was labelled with rabbit anti-adenovirus antibody (Abcam) followed by antirabbit immunoglobulin $\mathrm{G}$ antibody conjugated alkaline phosphatase (Abcam) for $1 \mathrm{~h}$ each. Finally, PNPP substrate was added to quantify the surface immobilized virus.

\section{Heterogeneity evaluation by Sips isotherm adsorption model}

Sips isotherm adsorption is a modified model from the Langmuir isotherm adsorption model:

$$
I=\frac{I^{s a t}(b C)^{a}}{1+(b C)^{a}}
$$

where $I$ is the intensity of adsorption that can be the optical density of the substrate PNPP $\left(\mathrm{OD}_{405}\right)$ in this study, $I^{\text {sat }}$ is the intensity during saturation, $C$ is the concentration of biotinylated molecules, $b$ is the affinity of biotinylated molecules towards avidin, and $a$ is the heterogeneity index, which is the exponent of the equation. By logarithmic expression, the equation can be expressed as:

$$
\ln \left(\frac{I}{I^{s a t}-I}\right)=a \ln b+a \ln C
$$

The heterogeneity index, $a$, and the affinity factor, $b$, can be determined by plotting $\ln \left(I /\left(I^{\text {sat }}-I\right)\right)$ to $\ln C$. The slope of the least-squares regression line would be $a$ and the intercept would be $a \ln b$.

Most often, the association constant between proteins and ligands follows a Gaussian distribution. Heterogeneity is used to describe the deviation of the association constant distribution when the protein is immobilized on a solid substrate. Because the associate and dissociate behaviour between immobilized proteins and their ligands deviates from when they are in solution, this deviation is always accompanied by a higher heterogeneity. The nonuniform binding affinity distribution can be evaluated by the heterogeneity index, $a$, which should be between 0 and 1 . When $a=1$, the affinity of an immobilized protein to ligands is the same as it is in solution, and a lower value $a$ indicates an increasing heterogeneity. Therefore, by a heterogeneity index comparison, we could evaluate the level of immobilized protein affected by the conjugation reaction. Lower affinity constants and heterogeneity indexes indicate that immobilization introduces heterogeneity into the binding behaviour and thus diminishes ligand binding activity [25].

\section{Scanning electron microscopy (SEM) illustrates virus immobilization on chitosan surfaces}

Chitosan surfaces with immobilized AdLacZ by the VBAM and VBABM methods were washed with PBS to remove unbound adenovirus and fixed with 10\% glutaraldehyde in PBS for $1 \mathrm{~h}$. Subsequently, these samples were postfixed in $1 \%$ osmium tetroxide (Acros) for $1 \mathrm{~h}$. After two washes with distilled water, the samples were incubated at $-80^{\circ} \mathrm{C}$ for $2 \mathrm{~h}$ and then lyophilized in a freeze dryer (Virtis, Gardiner, NY, USA) at $-78.51^{\circ} \mathrm{C}$ and $100 \mathrm{mTorr}$ for $24 \mathrm{~h}$. Samples were gold coated (SPI, West Chester, PA, USA) and examined by SEM (Nova Dual Beam FIB/SEM; FEI, Hillsboro, OR, USA).

\section{In vitro cell infection to determine viral activity}

Cell infection was performed to determine the cell transduction efficiency of the immobilized virus. The fibroblast cell line C4 was cultured at a density of $2 \times$ $10^{5}$ cells/well in 24-well culture plates coated by chitosan. Four different systems were compared: (i) VBAM; (ii) VBABM; (iii) a system in which the cells were seeded on one day and then infected by suspend virus (Free V); and (iv) a system in which both virus and cells were mixed and then seeded into the wells (Free V \& Cell).

To determine the exact amount of viral particles immobilized on chitosan surfaces in the VBAM and VABAM systems, a sandwich ELISA was performed. After the virus was immobilized on the material surface, $100 \mu \mathrm{l}$ of supernatant was applied to goat anti-adenovirus antibody coated 96-microwell plates for 1 hour. The bound virus was labelled by first and second antibody, and then was detected by substrate PNPP, as previously described in the virus immobilization experiment. Finally, the amount of immobilized virus was determined by subtracting the supernatant virus from the total amount of virus present before the reaction.

For cell infections, C4 fibroblasts were infected with a range of virus concentrations $\left(0-1.6 \times 10^{9} \mathrm{pfu} /\right.$ well $)$ and were cultured for $48 \mathrm{~h}$. The transduction efficiencies of each group were determined by the expression of $\beta$-galactosidase, which was detected using a sandwich ELISA kit, as previously described. The data were normalized to the amount of surface viral particles. Additionally, transduced cells were illustrated by staining with 5-bromo-4-chloro-3-indoyl- $\beta$-D-galactopyranoside (X-gal) [10]. 


\section{Results}

\section{Adenovirus biotinylation does not alter viral bioactivity}

Biotinylation of virus by SulfoNHS-LC-Biotin has been reported to preserve the infectivity of the virus [23]. Therefore, to maximize the level of biotinylation of adenovirus, a sandwich ELISA was used to quantify adenoviral surface biotinylation. Viral biotinylation was saturated when the concentration of SulfoNHS-LC-Biotin exceeded $0.5 \mathrm{mg}$ per $10^{12}$ viral particles (Figure 1a). The HABA assay of biotinylated virus demonstrated that there were $6209 \pm 505$ biotin molecules per viral particle. This high level of biotinylation suggests that virus can be robustly immobilized on chitosan. The viral infection efficiencies of adenovirus before and after biotinylation were also compared by infecting C4 fibroblasts. $\beta$-galactosidase expression by infected cells was quantified by ELISA (Figure 1b). The expression of biotinylated AdLacZ was in the range $80-90 \%$ in the nonmodified virus group, suggesting that virus infectivity can be preserved after biotinylation.

\section{Avidin/biotin crosslinked by homobifunctional crosslinker have different binding effects}

Two different strategies for avidin immobilization to chitosan were developed to tether viruses for in situ gene therapy. Avidin was either directly conjugated to a material surface (VBAM) or indirectly docked on surface conjugated biotin (VBABM). To optimize the crosslinker concentration of these two immobilization strategies, avidin and biotin were conjugated to chitosan surfaces with different concentrations of the homobifunctional glutaraldehyde crosslinker. These conjugates were analysed using biotin-AP and avidin-AP, respectively (Figures 2a and $2 \mathrm{~b}$ ). The avidin and biotin conjugation profiles differed significantly. Avidin conjugation increased with glutaraldehyde concentration and was saturated at $0.75 \%$ glutaraldehyde; however, a sharp decline in conjugation occurred with glutaraldehyde levels greater than $7.5 \%$ (Figure 2a). Biotin conjugation followed a different trend than avidin. The surface biotin, when in a system with increasing crosslinker, eventually reached a plateau at approximately $7.5 \%$ glutaraldehyde (Figure 2b). However, there was no decrease in conjugation at the highest concentration of crosslinker as was observed with avidin conjugation. Despite finding that $0.75 \%$ and $7.5 \%$ glutaraldehyde have the best conjugation rates for avidin and biotin, respectively, these high levels of glutaraldehyde may be harmful to cells. Therefore, to avoid potential cytotoxicity, $0.25 \%$ glutaraldehyde was applied for the following experiments because this level was effective for crosslinking and yet was nontoxic to cells in culture (data not shown). (a)
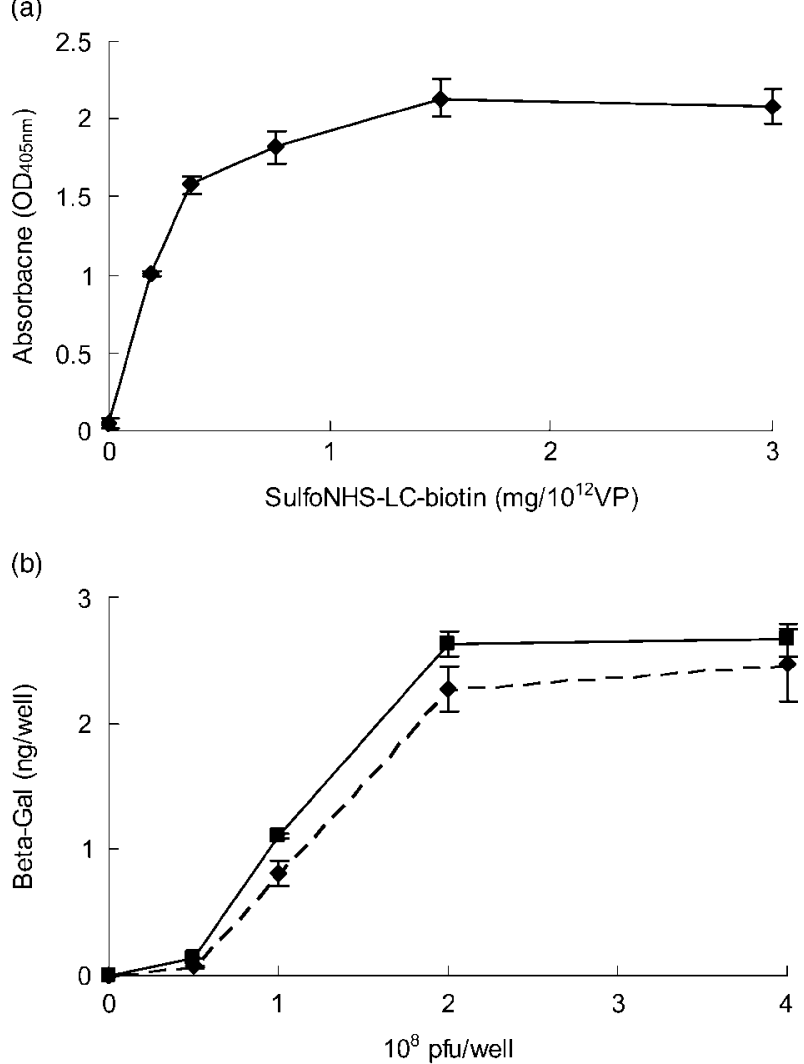

Figure 1. Adenovirus encoding LacZ can be biotinylated by SulfoNHS-LC-Biotin. (a) Biotinylated virus was immobilized on ELISA plates to detect and quantify biotin on viral surfaces using avidin-AP. The data suggest that biotinylation is dose-dependent and saturated when SulfoNHS-LC-Biotin is greater than $0.5 \mathrm{mg}$ per $10^{12}$ viral particles. (b) The $\beta$-galactosidase expression from cells infected by AdLacZ before (solid line) and after (dashed line) biotinylation were compared by sandwich ELISA. The results demonstrate that AdLacZ can maintain infection efficiency in the range $80-90 \%$ after biotinylation

Because high crosslinker concentrations led to a decrease in direct avidin conjugation, there may have been other factors affecting the crosslinking between avidin and material surfaces. To investigate this, avidin was conjugated to chitosan with different concentrations, in the range $1-250 \mu \mathrm{g} /$ well. Two experiments were performed to determine the amount of immobilized avidin molecules and the biotin binding sites, respectively. The avidin in the supernatant after the conjugation reaction was detected by UV spectrometry to indirectly quantify the immobilized avidin molecules on the material surface (Figure 3a). At the same time, the effective surface biotin binding sites on chitosan were directly determined with biotin-AP (Figure 3b).

From the UV detection assay, the immobilized avidin molecules on the chitosan surface increased with increasing concentrations of avidin. The conjugation efficiency was approximately $6.8 \%$ (Figure 3a). By contrast, when directly detecting surface binding sites on chitosan, high avidin concentrations increased biotin-AP immobilization; however, this trend plateaued with avidin concentrations greater than $30 \mu \mathrm{g} /$ well (Figure $3 \mathrm{~b}$ ). 
(a)

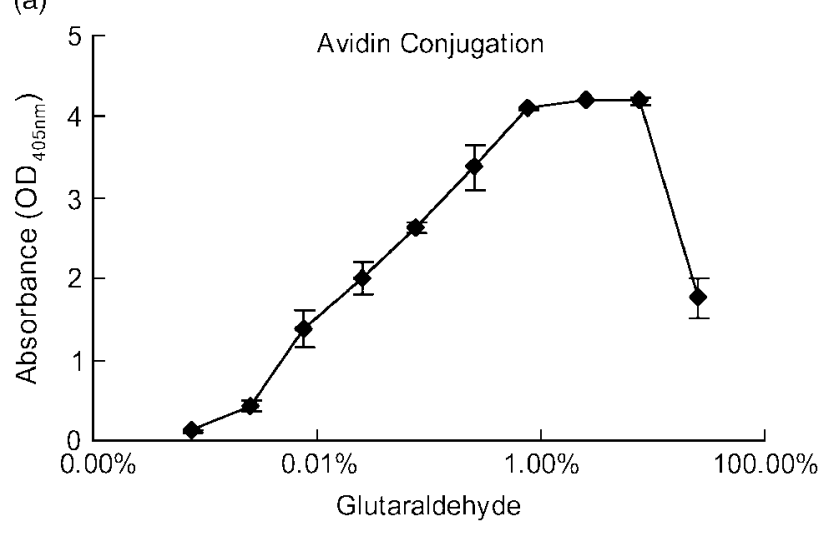

(b)

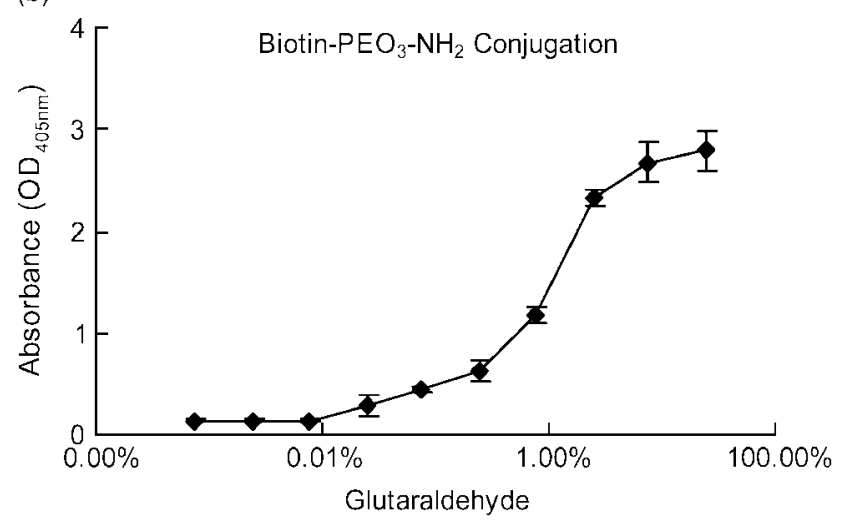

Figure 2. The conjugation profiles of homobifunctional crosslinker in the VBAM and VBABM systems are different. (a) In the VBAM system, avidin immobilized on chitosan increased with increasing concentrations of the crosslinker. However, extremely high crosslinker concentration led to a decrease in surface immobilization. (b) Because biotin conjugation by Amine- $\mathrm{PEO}_{3}-\mathrm{Biotin}$ can avoid self-crosslinking due to a single reactive amine group, the conjugation remained strong at saturation levels even in high crosslinker concentrations

\section{Comparison of VBAM and VBABM immobilization strategies}

To demonstrate differences in biotin immobilization efficiency between the VBAM and VBABM systems, biotin-AP and biotinylated adenovirus were compared (Figures 4a and 4b). Biotin-AP is a small protein whereas biotinylated adenovirus is a large complex, and thus they can be used to compare different immobilization effects for small and large biotinylated molecules. The surface immobilized avidin (30 $\mu \mathrm{g} /$ well) was used in both groups according to the saturation concentration from the prior titration experiment.

Results of the biotin-AP assay demonstrated that immobilization in both systems increased with biotinAP concentration (Figure 4a). To investigate the affinities of surface avidin to biotinylated molecules in these two systems, we referred to the Sips isotherm adsorption model because it is an association-dissociation assessment for antibody-antigen that is similar to biotin-avidin interaction $[25,26]$. The binding data were fitted into the Sips isotherm adsorption equation and the fitting
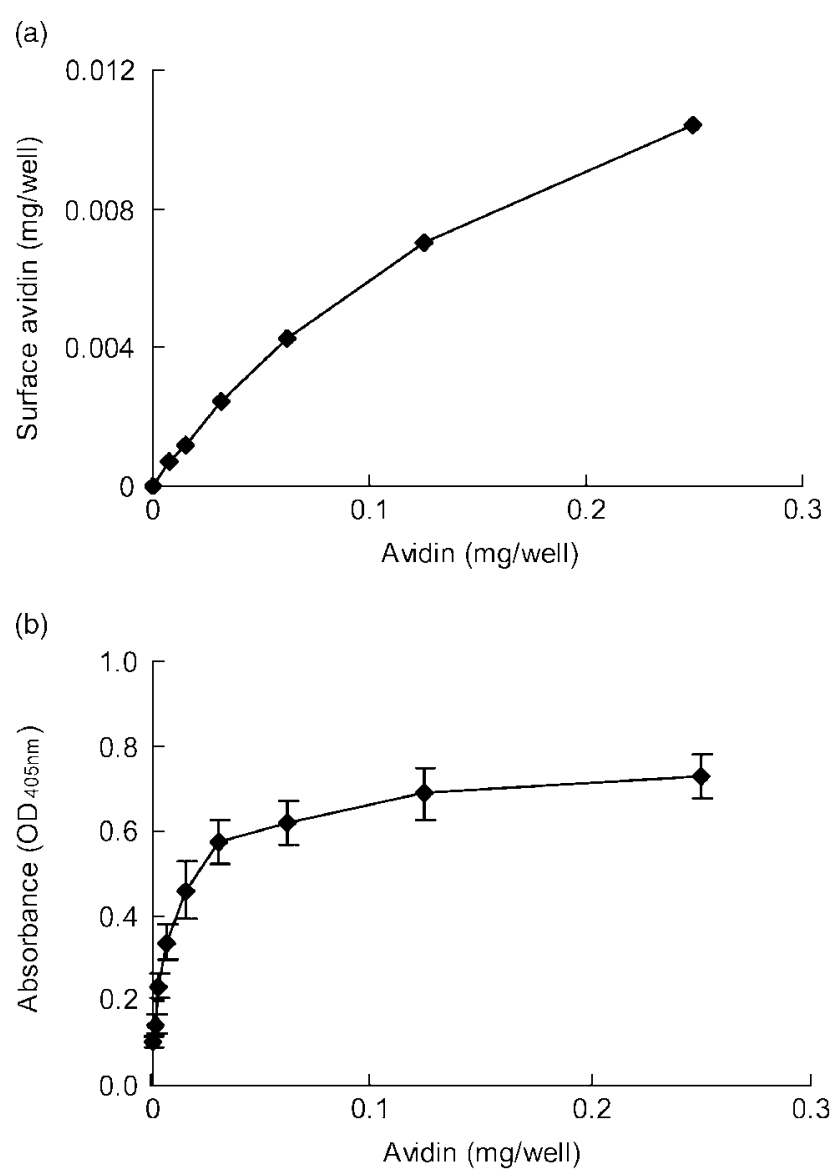

Figure 3. Avidin is conjugated on chiotsan as a multilayer. (a) Immobilized avidin molecules were evaluated by indirectly detecting unbound avidin suspended after conjugation. Immobilized avidin increased with increasing avidin concentration. (b) By contrast, biotin-AP analysis suggested that the surface binding sites reached a plateau when avidin concentrations were greater than $0.03 \mathrm{mg} /$ well, which is due to limited chitosan surfaces. The inconsistent trends suggest that a multilayer of avidin formed on the chitosan surface

parameters are summarized in Table 1 . The heterogeneity index, $a$, of VBABM was 1.000 , suggesting that the interaction between immobilized avidin and biotinAP was homogeneous. That is, the affinity of surface avidin for biotin-AP was similar to the affinity of avidin for biotin in solution. By contrast, the directly bound avidin in the VBAM system exhibited some heterogeneity ( $a=0.894$ ). The loading capacity in the VBABM system was also higher than in the VBAM system. The saturation intensities of bound alkaline phosphatase were 4.2 and 3.0 for VBABM and VBAM, respectively. Therefore, the lower heterogeneity index suggests that surface immobilization of the VBAM system induced heterogeneity between biotin-AP and avidin, whereas the VBABM system did not. This also suggests a diminished binding activity of the VBAM system.

For biotinylated virus immobilization, the trends of heterogeneity were more pronounced than those of biotinylated-AP for both the VBAM and VBABM methods (Figure 4b). The heterogeneity index of the VBAM system 

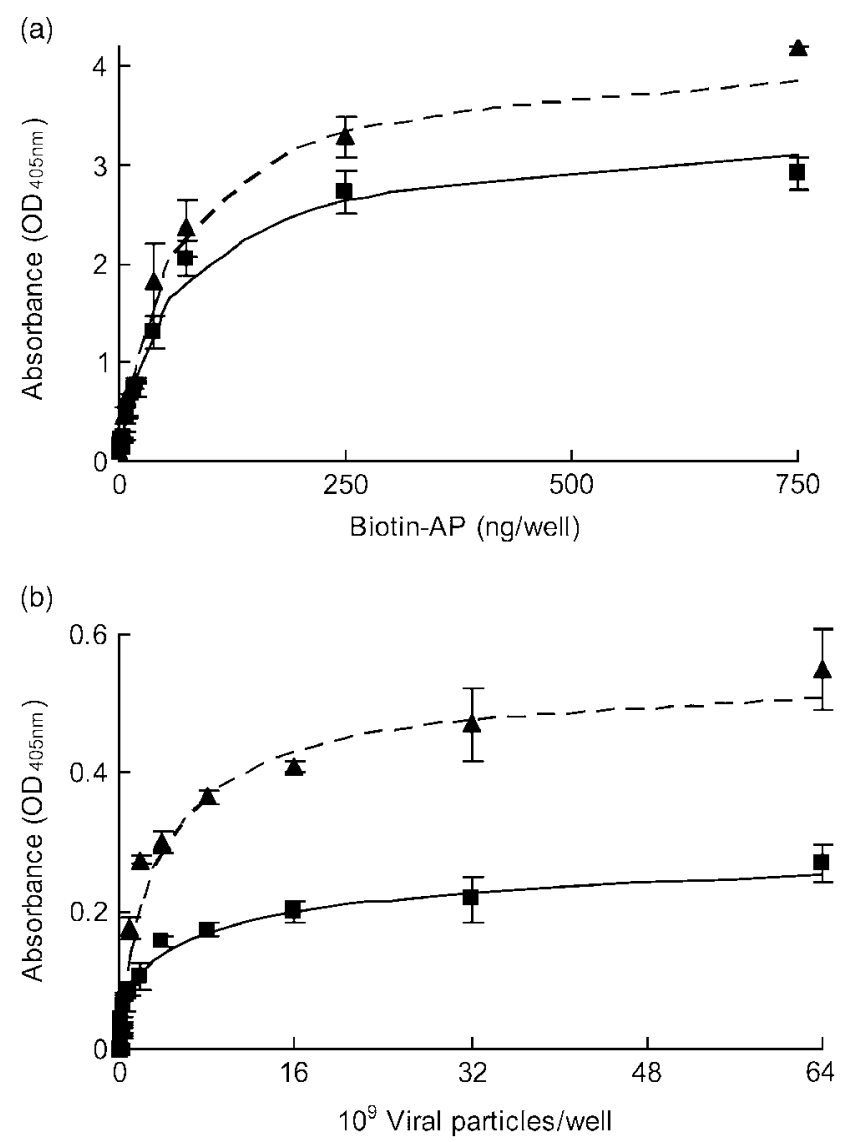

Figure 4. Biotinylated molecule immobilization of the VBABM system is greater than that of the VBAM system. Two different biotinylated molecules were applied and the binding capacities were compared: (a) biotin-AP and (b) biotinylated AdLacZ. The dashed and solid lines represent the Sips model fits of the data of VBABM (triangle) and VBAM (square) systems, respectively

( $a=0.540)$ was less than the VBABM system $(a=0.832)$. The saturation intensity of $\operatorname{VBAM}\left(I^{\text {sat }}=0.33\right)$ was also lower than VBABM $\left(I^{\text {sat }}=0.56\right)$. Moreover, the binding affinity of VBABM was almost double that of VBAM $\left(0.269 \mathrm{ml} / 10^{9} \mathrm{VP}\right.$ versus $\left.0.135 \mathrm{ml} / 10^{9} \mathrm{VP}\right)$ (Table 1). The lower heterogeneity indexes of both the VBAM and VBABM systems indicate that large biotinylated molecules, such as adenoviruses, may be more sensitive to steric hindrance than smaller molecules. This phenomenon of higher heterogeneity would affect the interaction between ligand (biotin) and receptor (avidin), and thus the adsorption behaviour would diverge from their homogeneous interaction in solution form.

\section{Immobilized virus distribution was examined by SEM}

SEM examination was performed to illustrate the surface virus distribution. In the VBAM system, virus clusters were found in low magnification (Figure 5a). These clusters were formed by uniform size particles, which were more obvious in high magnification. Compared to the typical sizes of adenovirus $(70-90 \mathrm{~nm})$, these particles, with diameter in the range $70-80 \mathrm{~nm}$, suggest that they are adenoviruses immobilized on chitosan surfaces [27].

Similarly, the VBABM system had viral particles the same size as those in the VBAM system. However, the distribution was different (Figure 5b). Evenly distributed particles without clusters were observed in this group. Moreover, the number of adenoviral particles bound in the VBABM group per unit area was obviously higher than in the VBAM group. These results suggest that biotinylated adenovirus can be more effectively immobilized by the VBABM method due to surface avidin alignment.

\section{VBABM system has an improved cell transduction efficiency over the VBAM system}

Adenovirus encoding the LacZ gene (AdLacZ) was used to transduce C4 fibroblasts to compare viral infection efficiencies of two immobilization strategies. The cells were infected and incubated for two days, followed by an ELISA assay to quantify $\beta$-galactosidase expression levels (Figure 6a). The adherent cells infected with free virus (Free V) had the lowest transduction rate. This rate was improved when cells were infected with virus in a suspended solution (Free V \& Cells) because the virus likely contacted cells in a homogeneous medium rather than in a potentially heterogeneous liquid-solid environment. Virus immobilized by the VBAM system was not superior because it had a similar infection profile to the Free $\mathrm{V}$ group. However, VBABM exhibited a significantly higher transduction efficiency. Moreover, the $\beta$-galactosidase activity at saturation in the VBABM group was $3 \mu \mathrm{g} /$ well compared to $2 \mu \mathrm{g} /$ well in the Free $\mathrm{V}$ and VBAM group and $2.5 \mu \mathrm{g}$ /well in the Free V \& Cell group. These findings suggest that biotin on biomaterial surfaces can increase the effective binding sites for biotinylated virus immobilization and thus enhance the transduction efficiency. The X-gal staining also illustrated that cells cultured on plates can be infected by VBAM and VBABM

Table 1. Fitting parameters for the Sips isotherm adsorption model

\begin{tabular}{lccccc}
\hline & \multicolumn{2}{c}{ Biotin-AP } & & \multicolumn{2}{c}{ Biotinylated AdLacZ } \\
\cline { 2 - 3 } \cline { 6 - 6 } & \multicolumn{1}{c}{ VBAM } & VBABM & & VBAM & VBABM \\
\hline Saturation intensity, / sat & 3.0 & 4.2 & & 0.33 & 0.56 \\
Heterogeneity index, $a$ & 0.894 & 1.000 & & 0.540 & 0.832 \\
Binding affinity $b$ & $14.1 \mathrm{ml} / \mathrm{pg}$ & $15.9 \mathrm{ml} / \mathrm{pg}$ & & $0.135 \mathrm{ml} / 10^{9} \mathrm{VP}$ & $0.269 \mathrm{ml} / 10^{9} \mathrm{VP}$ \\
\hline
\end{tabular}

VP, Viral particles. 
(a)
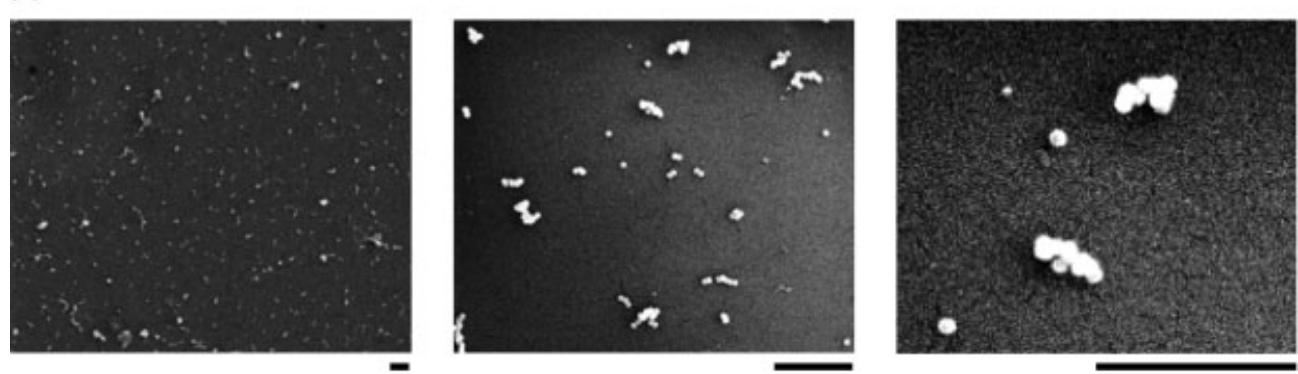

(b)
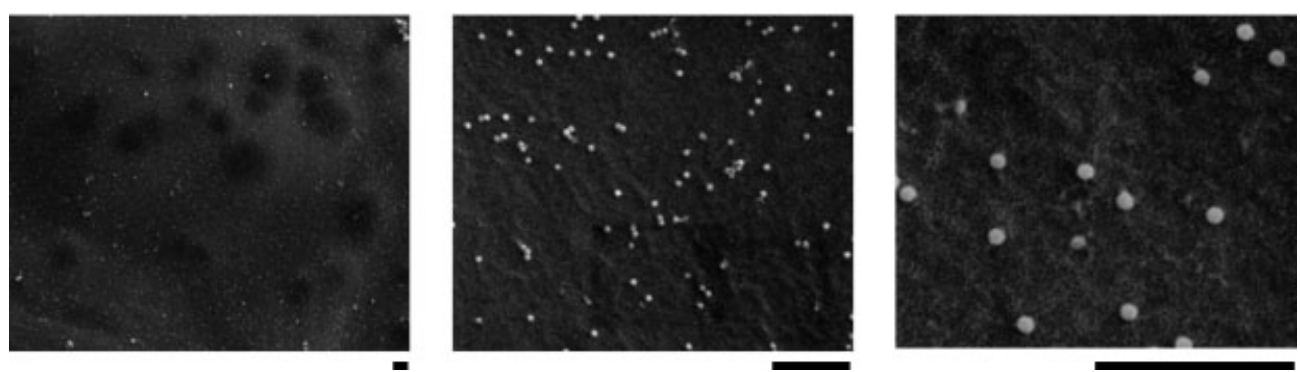

Figure 5. SEM images illustrate virus immobilization in both VBAM and VBABM systems. Adenovirus immobilized on chitosan surfaces was demonstrated by SEM examination. The chitosan surface images of (a) VBAM and (b) VBABM groups are shown with different magnifications. The scale bars under each picture are $1 \mu \mathrm{m}$. Viral particles tended to form aggregates or clusters in the VBAM system, whereas the VBABM system led to evenly distributed viral particles

systems and the $\beta$-galactosidase expression levels were consistent to their ELISA results (Figure 7).

The levels of virus in the VBAM and VBABM systems are likely underestimated because immobilized virus was not totally bound on the chitosan surfaces. Excess virus was removed by PBS washes prior to cell infection. The true amounts of immobilized virus were indirectly detected by quantifying unbound virus using a sandwich ELISA assay. In the VBABM group, $95-100 \%$ of adenovirus can be immobilized on surfaces when there are less than $1 \times 10^{8}$ viral particles per well. Higher virus concentration caused the immobilization rate to decline due to limited substrate area. In contrast, VBAM had only a $40-60 \%$ immobilization rate in the same concentration. Therefore, we normalized the cell transduction results to the immobilized virus levels. This normalization demonstrated better performances in both VBAM and VBABM systems. The result of the VBAM group was similar to the Free V \& Cell group, whereas the VBABM group had a much better performance than the other three groups (Figure 6b). $\beta$-galactosidase expression of VBABM was saturated when there were $4 \times 10^{7}$ pfu AdLacZ, which was almost six, ten and 50-fold greater than that of the VBAM, Free V \& Cell, and Free V groups, respectively, when using the same virus concentrations.

\section{Discussion}

The method of virus delivery can have a profound influence on the outcomes of regenerative gene therapy. While bolus delivery requires high viral titers and provides little control over virus diffusion, spatially controlled viral infection may restrict viral transduction to only the target sites and limit systemic infection. Virus localization can also improve transduction efficiency, and thus the viral dosage may be reduced [10,28,29]. These effects can decrease potential risks and increase therapeutic safety. In this study, virus immobilization was performed by avidin/biotin interaction, the strongest known noncovalent bond, which should be able to tightly tether virus on material surfaces [20]. However, some research indicates that the cell transduction of biotinylated adenovirus tethered on avidin coated plates for in situ transduction is modest in comparison to free infection delivery [23]. This may be due to steric hindrance of active binding sites, that can be inactivated when they are close to the solid support surface [30]. In addition, the microenvironment of the surface and the potential conformational changes of immobilized proteins can lead to heterogeneous binding affinity and association/dissociation kinetics to their complementary ligands [31-33]. Therefore, determining how to reduce the heterogeneity of the binding force and how to preserve protein function are important issues for protein surface immobilization.

To reduce heterogeneity, orientated immobilization is an improved strategy because it can reduce the blockage of binding sites. For example, to orientate immunoglobulin $\mathrm{G}$ on support surfaces for immunosorption, the Fc regions are used for immobilization on solid supports by either conjugating with its carbohydrate residues or by mediating protein $\mathrm{A} / \mathrm{G}$ conjugation [34-36]. These strategies allow the Fab regions to be exposed and thus avoid steric hindrance due to random conjugation. These data suggest that orientated immobilization can reduce 
(a)

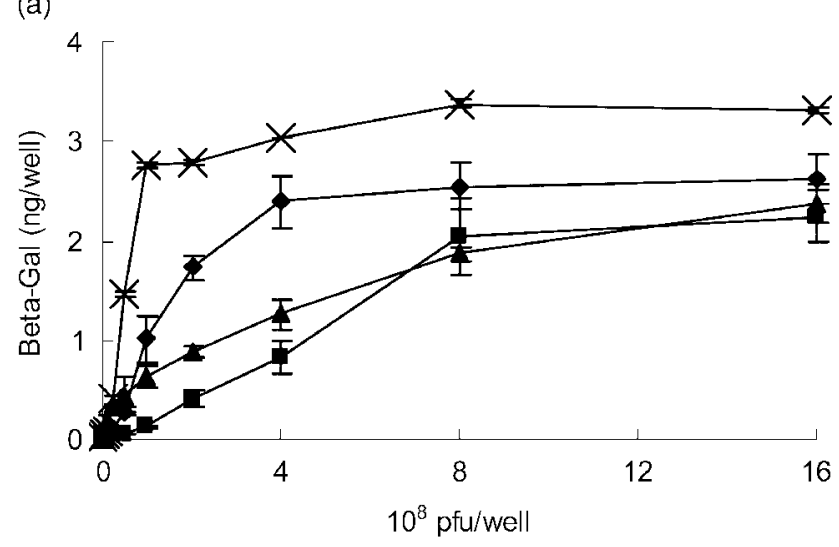

(b)

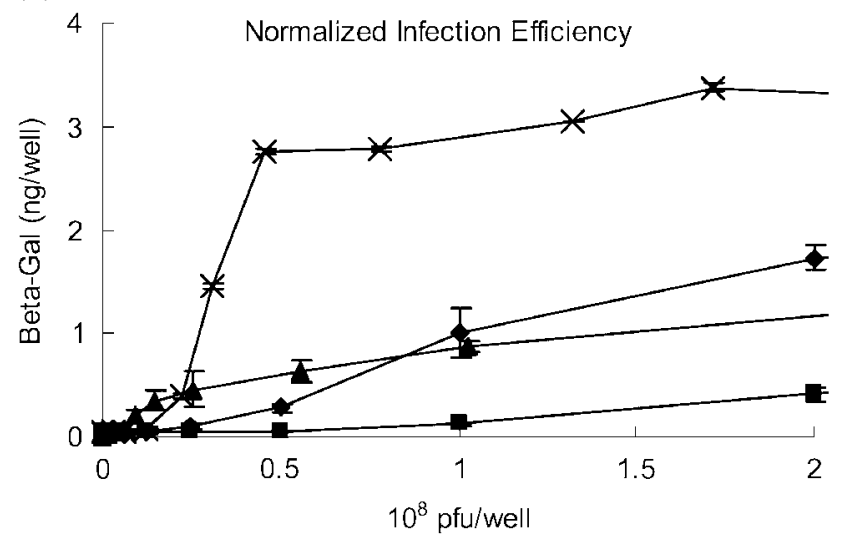

Figure 6. In vitro cell transduction demonstrates the infection efficiency of the immobilized virus. (a) Virus was immobilized by the VBAM (triangle) or VBABM (cross) methods on 24-well culture plates and then cultured with $2 \times 10^{5}$ cells/well. There were two control groups using suspended virus infection: (1) cells were plated for $24 \mathrm{~h}$ prior to infection in solution (Free V, square) and (2) cells and virus were mixed together before the cells were plated (Free V \& Cell, diamond). (b) The viral particles on chitosan surfaces were indirectly estimated by detecting unbound virus after immobilization. This reflects the real surface virus number and normalizes the virus infection result

heterogeneity and enhance receptor-ligand adsorption. By a similar hypothesis, one can assume that biotinylated virus immobilized on material surfaces can be orientated in the VBABM system because the immobilized avidin can be aligned by the surface conjugated biotin (Figure $8 \mathrm{~b}$ ). This differs from the randomly distributed avidin in the VBAM system due to nonspecific conjugation (Figure 8a).

In the present study, we used biotinylated reagent to modify viral capsid proteins. Compared to other research, that genetically fuses a biotin acceptor peptide to a virus and then biotinylates the virus later, our method is easier and can be applied more generally for different viral vectors [22]. The infectivity of biotinylated adenovirus can be maintained at $80-90 \%$, suggesting that SulfoNHS-LCBiotin is mild and appropriate for adenovirus modification (Figure 1b).

Conjugation with various crosslinker concentrations demonstrated different profiles of these two methods (Figures 2a and 2b). In the VBAM system, avidin was directly bound to the surface by glutaraldehyde, a homobifunctional crosslinker that randomly conjugates with amines to form Schiff bonds [37]. This conjugation not only links avidin to chitosan, but also to different avidins. Higher concentrations of glutaraldehyde can increase the crosslinking and thus immobilize more avidin on the material surface (Figure 2a). Saturation occurs due to the default area of chitosan coated on the wells. The amount of avidin may be increased with layering, but the total binding sites for biotin should be consistent. However, a concentration of crosslinker molecules that is too high may cause avidin conjugation to be distributed unevenly on the material surface. The increasing roughness may cause steric hindrance and thus decrease biotin immobilization. The multilayer property of the VBAM system was demonstrated by using different avidin concentrations to compare the relationship between surface avidin (UV detection) and potential binding sites (biotin-AP assay) (Figures $3 \mathrm{a}$ and $3 \mathrm{~b}$ ). The binding sites were increased with avidin concentration, and were saturated when avidin was greater than $30 \mu \mathrm{g} /$ well. By contrast, immobilized avidin kept rising even when the avidin concentration was more than $30 \mu \mathrm{g} /$ well. These results suggest that immobilization increases with increasing avidin concentrations during conjugation because of inter-avidin crosslinking, whereas the binding sites were limited due to the default area of the coated chitosan. Conversely, in the VBABM system, because the Amine- $\mathrm{PEO}_{3}$-Biotin has only one functional end for conjugation, multilayer formation such as in the VBAM system may not occur in this condition (Figure $2 \mathrm{~b}$ ).

Because avidin conjugation is nonspecific, biotin binding was likely affected to a certain extent by immobilization. Direct conjugation may inactivate some biotin binding sites on avidin. In addition, if the binding regions are close to the solid phase material or hindered by other avidin molecules, the affinity could be reduced because of steric blockage effects. To evaluate the effects of steric hindrance on affinities for different sized biotinylated molecules, biotin-AP and biotinylated AdLacZ were immobilized by surface avidin through the VBAM or VBABM methods. Binding affinity assessment was achieved by the heterogeneity index evaluation from the Sips isotherm adsorption model $[25,26]$. The saturation intensities for both biotin-AP and biotinylated AdLacZ indicated that the VBABM system had higher adsorption levels than VBAM (Figures 4a and 4b). Judging by Sips isotherm equations, VBABM expressed nearly homogeneous adsorption to biotin-AP. Even for large biotinylated molecules such as the biotinylated AdLacZ, the heterogeneity index of VBABM was closer to 1 than in the VBAM system (Table 1). This suggests that orientated avidin can enhance biotinylated molecule immobilization and reduce heterogeneity.

SEM illustrated that adenovirus was immobilized on material surfaces in both VBAM and VBABM groups because there were abundant uniformly sized particles on chitosan with diameters in the range $70-80 \mathrm{~nm}$, consistent with the typical diameters of adenovirus in 

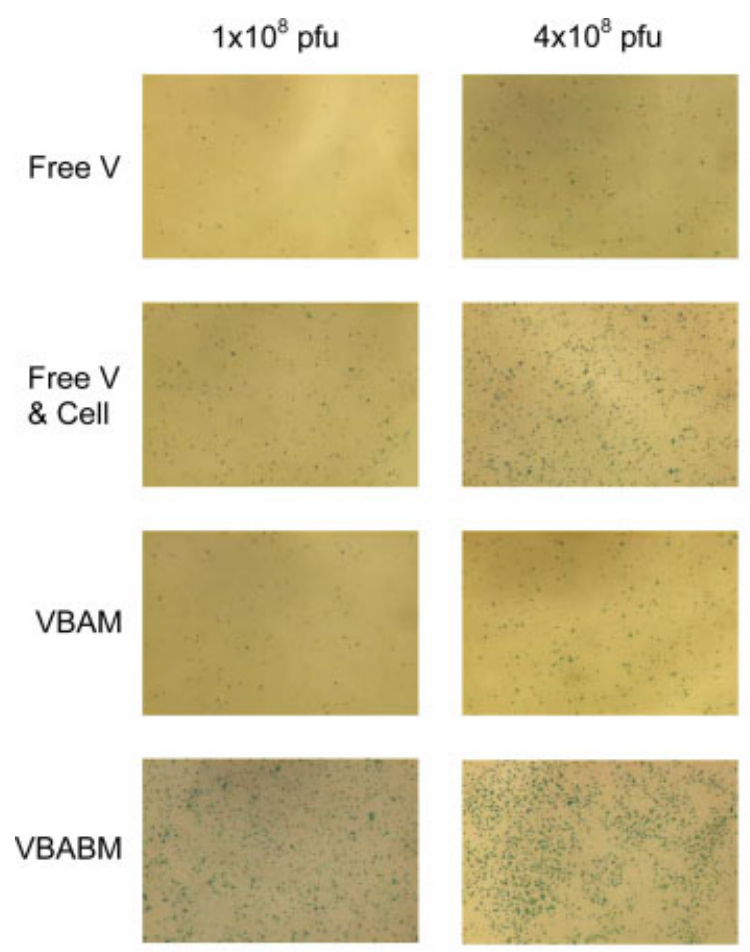
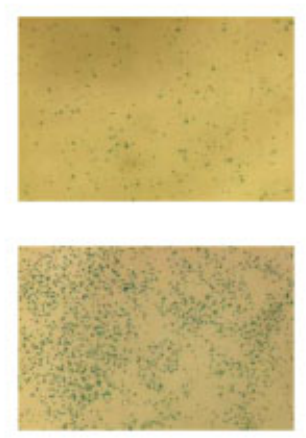
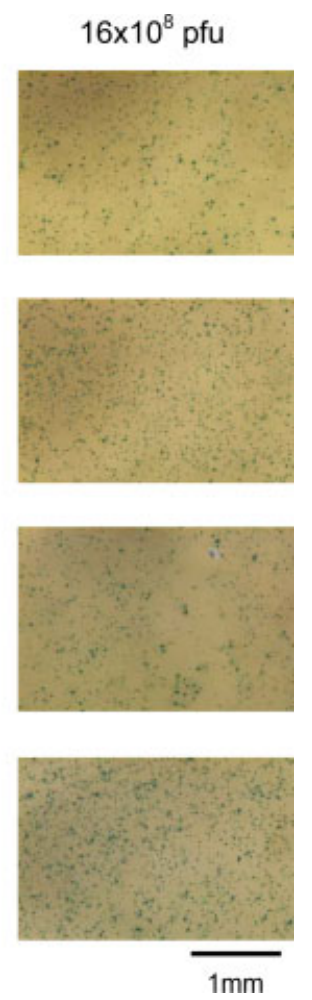

Figure 7. X-gal staining demonstrates $\beta$-galactosidase activity in infected cells using the four infection models and a range of viral particles $\left(1 \times 10^{8}\right.$ to $\left.16 \times 10^{8} \mathrm{pfu}\right)$. Darkly stained cells represent $\beta$-galactosidase expression in transduced cells. The VBABM system performed much better than the other groups and had maximal activity with as little as $1 \times 10^{8} \mathrm{pfu}$

(a)

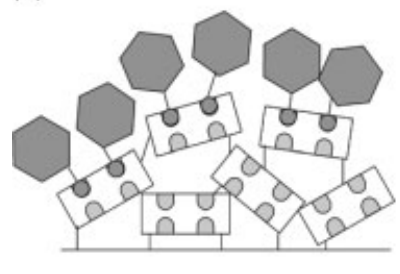

VBAM system (b)

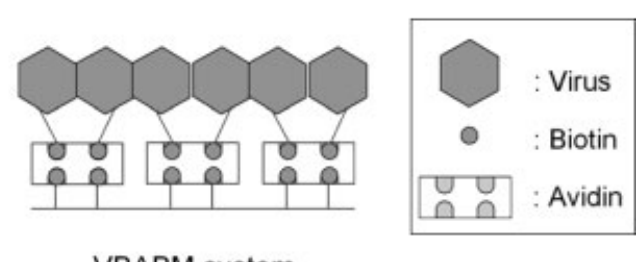

VBABM system

Figure 8. Schematic models of the two virus immobilization systems developed in the present study. (a) Virus-biotin-avidin-material (VBAM) system. (b) Virus-biotin-avidin-biotin-material (VBABM) system

the range $70-90 \mathrm{~nm}$ (Figures $5 \mathrm{a}$ and $5 \mathrm{~b}$ ). There were many aggregates of the adenovirus shown in the VBAM group, which is likely due to the unevenly distributed biotin binding sites (Figure 5a). In the VBAM method, random avidin conjugation may cause different avidin molecules to connect to each other. This inter-avidin crosslinking could lead to avidin being immobilized on the chitosan surface as clusters. By contrast, the VBABM system could avoid this drawback and, consequently, evenly distribute adenovirus on chitosan surfaces (Figure 5b).

In assessing in vitro cell transduction, the virus immobilized by the VBAM method demonstrated modest infection efficiency compared to the excellent transduction in the VBABM method. To fairly evaluate virus dosage in the VBAM and VBABM groups, suspended viral particles after conjugation were detected for normalization. The infection of VBAM was comparable to the Free V \& Cell group and was better than the Free $\mathrm{V}$ group after normalization (Figure 6b). Interestingly, VBAM still had a lower transduction efficiency than VBABM, even with the same number of immobilized viral particles. This may be due to the virus biotinylation reagent, SulfoNHS-LC-Biotin, which has a spacer between biotin and the viral protein. Because avidin immobilized by random conjugation may increase surface roughness and result in unevenly distributed binding sites, the spacer may be entangled when biotin is bound to the surface. This may inhibit a viral particle from being internalized into cells and thus reduce infection. Therefore, the orientated biotin immobilization in the VBABM strategy not only increases effective binding sites for biotinylated virus immobilization, but also improves virus infection efficiency.

Consequently, in the present study, a new method was developed to immobilize adenovirus on biomaterial surfaces for in situ gene therapy. Chitosan surfaces can be 
modified and used as an efficient viral carrier. In addition, adenovirus biotinylation can modify viral surfaces while preserving its infectivity. The VBABM model can evenly tether adenovirus on a chitosan surface, and can improve virus transduction. Excessive viral titer, which may induce cytotoxicity and unwanted systemic infection, may be avoided with this system. This model of viral delivery could be adapted for use with not only a variety of biomaterials, but also different types of viral vectors and thus may be an alternative method for in vivo regenerative gene therapy.

\section{Acknowledgements}

The research was supported by a grant from the AO Foundation (16-2004).

\section{References}

1. Pickles RJ, McCarty D, Matsui H, et al. Limited entry of adenovirus vectors into well-differentiated airway epithelium is responsible for inefficient gene transfer. J Virol 1998; 72: 6014-6023.

2. Alden TD, Pittman DD, Hankins GR, et al. In vivo endochondral bone formation using a bone morphogenetic protein 2 adenoviral vector. Hum Gene Ther 1999; 10: 2245-2253.

3. Musgrave DS, Bosch P, Ghivizzani S, et al. Adenovirus-mediated direct gene therapy with bone morphogenetic protein-2 produces bone. Bone 1999; 24: 541-547.

4. Okubo Y, Bessho K, Fujimura K, Iizuka T, Miyatake SI. Osteoinduction by bone morphogenetic protein-2 via adenoviral vector under transient immunosuppression. Biochem Biophys Res Commun 2000; 267: 382-387.

5. Kass-Eisler A, Falck-Pedersen E, Elfenbein DH, et al. The impact of developmental stage, route of administration and the immune system on adenovirus-mediated gene transfer. Gene Ther 1994; 1: $395-402$.

6. Ochiya T, Nagahara S, Sano A, Itoh H, Terada M. Biomaterials for gene delivery: atelocollagen-mediated controlled release of molecular medicines. Curr Gene Ther 2001; 1: 31-52.

7. Beer SJ, Matthews CB, Stein CS, et al. Poly (lactic-glycolic) acid copolymer encapsulation of recombinant adenovirus reduces immunogenicity in vivo. Gene Ther 1998; 5: 740-746.

8. Jang JH, Houchin TL, Shea LD. Gene delivery from polymer scaffolds for tissue engineering. Expert Rev Med Devices 2004; 1: 127-138.

9. Bengali Z, Rea JC, Shea LD. Gene expression and internalization following vector adsorption to immobilized proteins: dependence on protein identity and density. J Gene Med 2007; 9: $668-678$.

10. Hu WW, Wang Z, Hollister SJ, Krebsbach PH. Localized viral vector delivery to enhance in situ regenerative gene therapy. Gene Ther 2007; 14: 891-901.

11. Onishi H, Machida Y. Biodegradation and distribution of watersoluble chitosan in mice. Biomaterials 1999; 20: 175-182.

12. Rao SB, Sharma CP. Use of chitosan as a biomaterial: studies on its safety and hemostatic potential. J Biomed Mater Res 1997; 34: $21-28$.

13. Aspden TJ, Mason JD, Jones NS, et al. Chitosan as a nasal delivery system: the effect of chitosan solutions on in vitro and in vivo mucociliary transport rates in human turbinates and volunteers. J Pharm Sci 1997; 86: 509-513.

14. Suh JK, Matthew HW. Application of chitosan-based polysaccharide biomaterials in cartilage tissue engineering: a review. Biomaterials 2000; 21: 2589-2598.
15. Zhang Y, Zhang M. Calcium phosphate/chitosan composite scaffolds for controlled in vitro antibiotic drug release. $J$ Biomed Mater Res 2002; 62: 378-386.

16. Li Z, Ramay HR, Hauch KD, Xiao D, Zhang M. Chitosan-alginate hybrid scaffolds for bone tissue engineering. Biomaterials 2005; 26: 3919-3928.

17. Levy RJ, Song C, Tallapragada S, et al. Localized adenovirus gene delivery using antiviral IgG complexation. Gene Ther 2001; 8: 659-667.

18. Klugherz BD, Song C, DeFelice S, et al. Gene delivery to pig coronary arteries from stents carrying antibody-tethered adenovirus. Hum Gene Ther 2002; 13: 443-454.

19. Mei L, Jin X, Song C, Wang M, Levy RJ. Immobilization of gene vectors on polyurethane surfaces using a monoclonal antibody for localized gene delivery. J Gene Med 2006; 8: 690-698.

20. Green NM. Avidin. 1. The use of (14-C)biotin for kinetic studies and for assay. Biochem $J$ 1963; 89: 585-591.

21. Yao D, Vlessidis AG, Evmiridis NP. Microdialysis sampling and monitoring of uric acid in vivo by a chemiluminescence reaction and an enzyme on immobilized chitosan support membrane. Anal Chim Acta 2003; 478: 23-30.

22. Parrott MB, Adams KE, Mercier GT, et al. Metabolically biotinylated adenovirus for cell targeting, ligand screening, and vector purification. Mol Ther 2003; 8: 688-700.

23. Hobson DA, Pandori MW, Sano T. In situ transduction of target cells on solid surfaces by immobilized viral vectors. BMC Biotechnol 2003; 3: 4.

24. Nanci A, Wuest JD, Peru L, et al. Chemical modification of titanium surfaces for covalent attachment of biological molecules. J Biomed Mater Res 1998; 40: 324-335.

25. Vijayendran RA, Leckband DE. A quantitative assessment of heterogeneity for surface-immobilized proteins. Anal Chem 2001; 73: 471-480.

26. Sips R. On the structure of a catalyst surface. J Chem Phys 1948; 16: 490-495.

27. Dimmock NJ, Primrose SB (eds). Introduction to Modern Virology. Blackwell Press: London, 1994; 345.

28. Bajaj B, Lei P, Andreadis ST. High efficiencies of gene transfer with immobilized recombinant retrovirus: kinetics and optimization. Biotechnol Prog 2001; 17: 587-596.

29. Hanenberg H, Xiao XL, Dilloo D, et al. Colocalization of retrovirus and target cells on specific fibronectin fragments increases genetic transduction of mammalian cells. Nat Med 1996; 2: 876-882.

30. Lu B, Smyth MR, O'Kennedy R. Oriented immobilization of antibodies and its applications in immunoassays and immunosensors. Analyst 1996; 121: 29R-32R.

31. Yeung C, Leckband D. Molecular level characterization of microenvironmental influences on the properties of immobilized proteins. Langmuir 1997; 13: 6746-6754.

32. Yeung C, Purves T, Kloss AA, et al. Cytochrome c recognition of immobilized, orientational variants of cytochrome $b(5)$ : direct force and equilibrium binding measurements. Langmuir 1999; 15: 6829-6836.

33. Firestone MA, Shank ML, Sligar SG, Bohn PW. Film architecture in biomolecular assemblies. Effect of linker on the orientation of genetically engineered surface-bound proteins. J Am Chem Soc 1996; 118: 9033-9041.

34. Shriver-Lake LC, Donner B, Edelstein R, et al. Antibody immobilization using heterobifunctional crosslinkers. Biosens Bioelectron 1997; 12: 1101-1106.

35. Anderson GP, Jacoby MA, Ligler FS, King KD. Effectiveness of protein A for antibody immobilization for a fiber optic biosensor. Biosens Bioelectron 1997; 12: 329-336.

36. Lu B, Smyth MR, Okennedy R. Immunological activities of IgG antibody on pre-coated Fc receptor surfaces. Anal Chim Acta 1996; 331: 97-102.

37. Ahmed SR, Kelly AB, Barbari TA. Controlling the orientation of immobilized proteins on an affinity membrane through chelation of a histidine tag to a chitosan-Ni ${ }^{++}$surface. J Memb Sci 2006; 280: $553-559$. 